\title{
Provenance Provisioning in Mobile Agent-Based Distributed Job Workflow Execution
}

\author{
Yuhong Feng and Wentong Cai \\ School of Computer Engineering, Nanyang Technological University \\ Singapore 639798 \\ \{YHFeng, ASWTCai\}@ntu.edu.sg
}

\begin{abstract}
Job workflow systems automate the execution of scientific applications, however they may hide how the results are achieved (i.e., the provenance information of the job workflow execution). This paper describes the development and evaluation of a decentralized recording and collection scheme for job workflow provenance in mobile agent-based distributed job workflow execution. A performance study was conducted to evaluate our approach against the one using a centralized provenance server. The results are discussed in the paper.
\end{abstract}

Keywords: Distributed Job Workflow Execution, Provenance Recording and Collection, Grid Computing.

\section{Introduction}

The provenance of some data is defined as the documentation of the process that led to the data [1]. The necessity of provenance for job workflow execution is apparent since provenance provides a traceable path on how a job workflow was executed and how the resulted data were derived. It is particularly important in Service Oriented Architecture (SOA) since shared services and data sets might be used in the course of the job workflow execution. Provenance information can be processed and used for various purposes, for example, for validation of e-Science experiments [2], credibility analysis of the results of workflow execution [3], faulttolerance for service-based applications [4, and data sets regeneration for data intensive scientific applications [5].

The provenance information can be generated from the static information available in the original workflow specification (e.g., data dependencies) together with the runtime details obtained by tracing the execution of the workflow execution. The trace can be automatically generated by developing either a special "wrapping service" of the engine [6] or an "engine plugin" 7] to capture and record provenance related data directly from the workflow engine. The workflow trace can also be collected collectively by the services that execute the subjobs [8] or the services together with the workflow engine [9]. But, this puts the responsibility of provenance data recording to the service providers and may also require service modification. 
No matter how the traces are collected, in general some special provenance services are used in the current systems to store the provenance data and to provide an interface for users to query the data. Thus, a protocol is needed for various service providers and the workflow engine to communicate with the provenance services during the provenance collection process [1. A taxonomy of data provenance techniques can be found in [10], and a comprehensive documentation on provenance architecture can be found in [11.

Data intensive scientific applications often involve high volume, and distributed data sets. They can be generally expressed as a workflow of a number of analysis modules, each of which acts on specific sets of data and performs cross multidisplinary computations. To reduce the communication overhead caused by data movement and to provide decentralized control of execution during workflow enactment, the Mobile Code Collaboration Framework (MCCF) is developed to map the execution of subjobs to the distributed resources and to coordinate the subjobs' execution on runtime according to the abstract workflow provided by users [12]. LMA [13] is used in the MCCF for the purpose of separating functional description and executable code. The functional description of LMA is described using Agent Core (AC). An AC is essentially an XML file, containing the job workflow specification and other necessary information for agent creation and execution. An AC can be migrated from one resource to another. As for the executable code, to separate subjob specific code and common non-functional code (i.e., code for handling resource selection, subjob execution, agent communication, and AC migration), Code-on-Demand (CoD) [14 is used in the MCCF, that is, subjob specific code is downloaded to the computational resource and executed on demand. This enables an analysis module in the data intensive scientific applications to be executed at a computational resource close to where the required data set is. The execution of common non-functional code is carried out by a group of underlying $A C$ agents (or agents in short).

The MCCF, which does not have a centralized engine, is different from the existing scientific workflow engines (e.g., Condor's DAGMan! and SCIRun 22). Hence, the objective of this paper is to develop a provenance recording and collection algorithm so that mobile agents deployed in the execution of a job workflow can collectively collect a complete set of information about the job workflow execution.

\section{Partner Identification in the MCCF}

Job workflows in MCCF are modeled using Directed Acyclic Graph (DAG). A DAG can be denoted as $\mathcal{G}=(\mathcal{J}, \mathcal{E})$, where $\mathcal{J}$ is the set of vertices representing subjobs, i.e., $\mathcal{J}=\left\{J_{0}, J_{1}, \ldots, J_{n-1}\right\}$. $\mathcal{E}$ is the set of directed edges between subjobs. There is a directed edge from subjob $J_{i}$ to $J_{j}$ if $J_{j}$ requires $J_{i}$ 's execution results as input. Data dependency (denoted as "<") between two subjobs exists if there

\footnotetext{
1 http://www.cs.wisc.edu/condor/dagman/

2 http://software.sci.utah.edu/scirun.html
} 


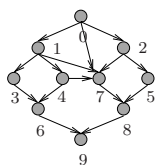

1. assign subjob ID

(a)

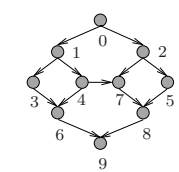

2. remove edges indicating indirect successors

(b)

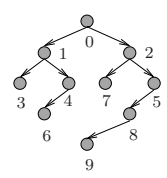

3. generate a spanning tree

(c)

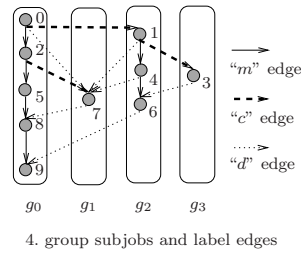

(d)

Fig. 1. Steps of the Preprocessing Algorithm

is a path between the subjobs. It is also assumed that a DAG representing a job workflow always has a unique staring node, $J_{0}$, and a unique end node, $J_{n-1}$.

When multiple data independent subjobs can be executed concurrently, replicas of an existing $\mathrm{AC}$ will be generated so that there is one $\mathrm{AC}$ for each subjob. MCCF uses a preprocessing algorithm to generate information for determining when and how to create $\mathrm{AC}$ replicas and generate information for the decision of which AC replica's agents will be responsible for the resource selection for a common successor [15]. The preprocessing algorithm performs the following steps: (i) Assign subjob ids: Topological order is used to assign subjob ids from 0 to $n-1$, as shown in Figure 1(a). (ii) Remove indirect successors. Let $\mathcal{S}\left(J_{i}\right)$ denote the successor set of subjob $J_{i} . \forall J_{j_{1}}, J_{j_{2}} \in \mathcal{S}\left(J_{i}\right), J_{j_{2}}$ is called $J_{i}$ 's indirect successor if $J_{j_{1}}<J_{j_{2}}$, otherwise, it is called $J_{i}$ 's immediate successor. If $J_{j}$ is the indirect successor of $J_{i}$, the corresponding edge $\left(J_{i}, J_{j}\right)$ will be removed from the DAG, as shown in Figure 1(b). (iii) Generate spanning tree: A spanning tree is generated based on a Depth-First Search (DFS) 3 on the DAG, as shown in Figure 1(c). (iv) Group subjobs: A pre-order traversal is applied to the spanning tree to generate a visit sequence of nodes, and the leaves in this sequence will partition it into a number of groups. For example, the visit sequence generated for the spanning tree shown in Figure 1(c), $<0,2,5,8,9,7,1,4,6,3>$, is partitioned into groups: $\{0,2,5,8,9\},\{7\},\{1,4,6\}$ and $\{3\}$, as illustrated in Figure1(d). (v) Label edges: The edges between two subjobs, $J_{i}$ and $J_{j}$, in the same group are labeled " $m$ " (denoted as $J_{i} \stackrel{m}{\rightarrow} J_{j}$ ), the edges between subjobs of different groups are labeled "c" (denoted as $J_{i} \stackrel{c}{\rightarrow} J_{j}$ ), and the edges in the original DAG but not in the spanning tree are labeled " $d$ " (denoted as $J_{i} \stackrel{d}{\rightarrow} J_{j}$ ), as illustrated in Figure 1(d).

The preprocessed information is included in the AC and used during the dynamic job workflow execution. Suppose the current subjob is $J_{i}$, for one of its outgoing edges, $\left(J_{i}, J_{j}\right)$ : (i) If $J_{i} \stackrel{m}{\rightarrow} J_{j}$, the $\mathrm{AC}$ agents will select the resources for executing $J_{j}$. The AC replica will be migrated for $J_{j}$ 's execution after $J_{i}$ completes its execution. (ii) If $J_{i} \stackrel{c}{\rightarrow} J_{j}$, similar to the last case, the AC agents will select the resources for executing $J_{j}$, but a new AC replica will be created

${ }^{3}$ We assume that the depth-first search and pre-order traversal algorithms visit child nodes in the order from right to left. 
for $J_{j}$ 's execution. (iii) If $J_{i} \stackrel{d}{\rightarrow} J_{j}$, the AC agents need to communicate so that $J_{j}$ can locate the location of $J_{i}$ 's execution result.

The MCCF uses a contact list based agent communication mechanism [16] for subjob result notification. Two agents communicating with each other are called communication partners (or partners in short). Each AC maintains a list of partners with their locations (that is, a contact list). Before an AC is migrated or discarded, its agents will notify its partners so that they can update their contact lists accordingly. The location of the subjob's execution result will also be notified to the partners. The partner identification is carried out dynamically during the job workflow execution. Assume $\mathcal{E}_{d}$ is a set of edges that are marked with " $d$ " in $\mathcal{G}=(\mathcal{J}, \mathcal{E})$. Also assume that in the spanning tree generated by the preprocessing algorithm described above, a sub-tree rooted at $J_{i}$ is denoted as $\mathcal{T}_{J_{i}}$. Suppose $J_{i}$ and $J_{j}$ are two subjobs that are currently under execution, if $\exists\left(J_{i^{\prime}}, J_{j^{\prime}}\right) \in \mathcal{E}_{d}$, and $J_{i^{\prime}} \in \mathcal{T}_{J_{i}} \wedge J_{j^{\prime}} \in \mathcal{T}_{J_{j}}$, then the AC agents executing the subjobs $J_{i}$ and $J_{j}$ are partners 4 .

\section{Provenance Recording and Collection Protocol}

Let $G\left(J_{i}\right)$ be the group id of subjob $J_{i}$. It is easy to prove that the grouping algorithm described in the last section has the following property:

In $\mathcal{G}, \forall J_{i} \in \mathcal{J}$ and $G\left(J_{i}\right)>0$, there exists a path $\mathrm{p}$ from $J_{i}$ to $J_{n-1}$, where for any two consecutive subjobs, e.g., $J_{k_{q}}$ and $J_{k_{q+1}}$, on the path p, we have $G\left(J_{k_{q}}\right) \geq G\left(J_{k_{q+1}}\right)$.

$\mathrm{p}$ is called a propagation path. It is obvious that the $\mathrm{AC}$ that is finally returned to the user (that is, the original AC created by the user) will contain a complete provenance information for the job workflow if partners with higher group id propagate provenance information to the partners with the lower group ids during the job workflow execution. This forms the basis for the development of the provenance recording and collection protocol.

Let $\mathcal{R}\left(J_{i}\right)$ denote a subset of $J_{i}$ 's successors, where for any subjob $J_{j} \in \mathcal{R}\left(J_{i}\right)$, we have either $\left(J_{i} \stackrel{m}{\rightarrow} J_{j}\right)$ or $\left(J_{i} \stackrel{c}{\rightarrow} J_{j}\right)$. Assuming that subjob $J_{i}$ is under execution, the main steps of the protocol are:

- On receiving a communication message from its partner, $J_{i}$ updates its AC to include the provenance information and updates its contact list accordingly.

- On completion of $J_{i}$ 's execution, $J_{i}$ 's corresponding AC agents will record the location of $J_{i}$ 's execution result into $J_{i}$ 's AC.

- If $\mathcal{R}\left(J_{i}\right) \neq \emptyset$, as stated in Section 2, for each subjob $J_{j}, J_{j} \in \mathcal{R}\left(J_{i}\right), \mathrm{AC}$ agents corresponding to $J_{i}$ will locate resources, that is, the computational resource, the input data sets from the distributed data repository, and the code from the code repository, for the execution of $J_{j}$. These information

${ }^{4}$ More detail about the preprocessing based dynamic partner identification algorithm can be found in 15 . 
will be recorded in $J_{i}$ 's AC. Then, if $\left|\mathcal{R}\left(J_{i}\right)\right|>1,\left|\mathcal{R}\left(J_{i}\right)\right|-1$ replicas of $J_{i}$ 's AC will be created, one for each subjob $J_{j}, J_{j} \in \mathcal{R}\left(J_{i}\right)$ and $\left(J_{i} \stackrel{c}{\rightarrow} J_{j}\right)$.

- Before $J_{i}$ 's corresponding AC is migrated (or discarded if $\mathcal{R}\left(J_{i}\right)=\emptyset$ ), $J_{i}$ 's $\mathrm{AC}$ agents will send a communication message to all its partners in the contact list for execution coordination and contact list updating. The message contains the location of $J_{i}$ 's execution result, and the scheduling information for each $J_{j}, J_{j} \in \mathcal{R}\left(J_{i}\right)$. The scheduling information for subjob $J_{j}$ includes: subjob id, the id of the AC replica to be used to execute the subjob, locations of the selected computational resource, input data sets, and code for the execution of the subjob. In addition, if the partner has a smaller group id, provenance information received by $J_{i}$ from its partners with a larger group id (recorded in $J_{i}$ 's AC replica) during $J_{i}$ 's execution is also piggyback on the message.

Using the above protocol, the provenance information will be recorded in the $\mathrm{AC}$ replicas and propagated along propagation paths during the distributed execution of job workflow. Eventually, the AC that is finally returned to the user will contain the complete provenance information about the job workflow execution.

\section{Performance Evaluation}

As explained in the last section, the provenance information is transmitted along with the messages for execution coordination and contact list updating. Although there is no additional message required, the size of message will be increased. There is no centralized server used during the provenance information recording and collection.

Execution provenance information can also be collected using a centralized provenance server which maintains a provenance repository. For each subjob executed, the AC agents need to notify the centralized server about the provenance information. After a job workflow completes its execution, users can then get the provenance information from the server. In this centralized approach, additional messages are required for $\mathrm{AC}$ agents to communicate with the provenance server. Assuming that there is no need to collect provenance information for the start and end nodes (since they are assumed to have zero computation cost), the traffic generated in the centralized model can be estimated by:

$$
(n-2) * \overline{m s g}
$$

where $\overline{m s g}$ denotes the average size of a provenance message carrying a single provenance record.

To evaluate the performance of our distributed provenance collection algorithm, randomly generated Task Graphs (TG), that is, job workflows, were executed in the prototype MCCF system [12 on a cluster of computers. Six pseudo-random TGs were generated using TGFF5. As stated in Section $2 \forall J_{i} \in$

\footnotetext{
5 http://ziyang.ece.northwestern.edu/tgff/
} 

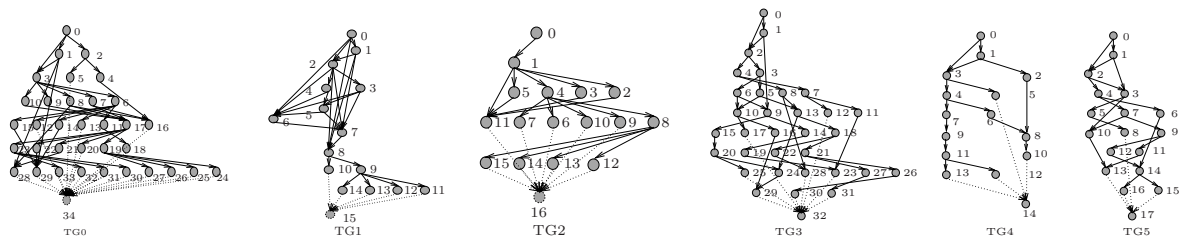

Fig. 2. Random Task Graphs

$\mathcal{J}, 0<i<(n-1), J_{0}<J_{i}<J_{n-1}$. Thus, when a TG has multiple subjobs that have no offspring, a hypothetical subjob is added, with no computation cost, to serve as the common immediate successor of such subjobs. The generated TGs are illustrated in Figure 2, where the dotted filled circles represent the added hypothetical subjobs, and the dotted edges represent the added edges.

The number of messages for execution coordination and contact list updating is illustrated in Table 1] These communication messages are required no matter whether decentralized or centralized method is used. During a job workflow execution, the messages that carry the propagated provenance records and the number of provenance records carried by such message were tracked. For a fair comparison with the centralized method, assuming that each propagated provenance record is carried by a separate provenance message, the total number of such messages in the decentralized method would be the summation of the number of propagated provenance records contained in all the tracked message 6 . Each job workflow was executed 3 times, and the average numbers are shown in Table 1. Note that for the centralized method, formula (11) is used to calculate the number of messages generated for the provenance collection.

Table 1. Experiment Results

\begin{tabular}{|c|c|c|c|c|}
\hline Task Graph & Msgs for Execution & \multicolumn{2}{|c|}{ Msgs for Provenance } & $\%$ \\
\cline { 3 - 4 } & Coordination & Improvement \\
\hline TG0 & 38 & 8 & 33 & $76 \%$ \\
\hline TG1 & 5 & 0 & 14 & $100 \%$ \\
\hline TG2 & 14 & 0 & 15 & $100 \%$ \\
\hline TG3 & 44 & 11 & 31 & $65 \%$ \\
\hline TG4 & 12 & 0 & 13 & $100 \%$ \\
\hline TG5 & 21 & 7 & 16 & $56 \%$ \\
\hline
\end{tabular}

Table 1 also shows the percentage improvement of the decentralized method over the centralized one. From these results, we observe that the centralized model always generates higher number of messages for provenance information recording. Particularly, by recording the provenance information in the workflow

${ }^{6}$ Note that in general the bandwidth consumed by two or more messages sent separately is larger than that of sending them in a single bundle [17. 
specification in the $\mathrm{AC}$, provenance record does not always needs to be propagated during a job workflows' execution. For example, no additional provenance record is propagated during the execution of task graphs TG1, TG2, and TG4 (and thus there is no provenance message required). In the execution of these task graphs, the agents of the AC replicas created during the runtime are the partners of the agents of the original AC. Therefore, propagation of provenance information is not required. In this case, the percentage improvement of the decentralized model over the centralized model is $100 \%$.

\section{Conclusion and Future Work}

Mobile agent-based distributed job workflow execution hides scientists from the Grid details, but also hides how the result is achieved (that is, the provenance of the job workflow execution). Since data processing in scientific computing may require some level of validation and verification, the information on the services and data sets used during the workflow execution is required. The provenance in many existing scientific workflow engines relies on a centralized provenance collection server for provenance recording and collection. However, in mobile agent-based distributed job workflow execution, there is no centralized workflow engine and thus naturally the provenance recording and collection should also be carried out in a distributed manner.

By studying the agent communication in the MCCF and the properties of the preprocessing algorithm for partner identification, a distributed provenance recording and collection protocol has been developed. The subjob provenance information is transmitted along the provenance propagation paths. Since provenance information is piggyback with the messages for execution coordination and contact list updating, there is no additional message required. To evaluate our approach, experimental study has been carried out on randomly generated job workflows. The results show that our approach has less communication overhead than the one using a centralized provenance server for provenance information recording and collection.

In the current algorithm, a subjob's provenance information might be propagated along multiple propagation paths. As a future work, the shortest and unique propagation path for a given subjob will be identified. This will further reduce the communication cost caused by propagation of provenance information. Although currently the execution coordination in the MCCF uses the contact list based mechanism, the provenance recording and collection protocol proposed in this paper should also work for other message-passing based execution coordination mechanisms (e.g., mailbox based mechanism [18]).

\section{References}

1. Groth, P., Luck, M., Moreau, L.: A protocol for recording provenance in serviceoriented grids. In: 8th Intl Conf on Principle of Distributed Systems (PODIS2004), Grenoble, France (December 2004) 124-139 
2. Wong, S.C., Miles, S., Fang, W.J., Groth, P., Moreau, L.: Provenance-based validation of e-science experiments. In: 4th Intl Semantic Web Conference. Volume 3729., Galway, Ireland (November 2005) 801-815

3. Rajbhandari, S., Wootten, I., Ali, A.S., Rana, O.F.: Evaluating provenance-based trust for scientific workflows. In: 6th IEEE Intl Symp on Cluster Computing and the Grid (CCGrid2006), Singapore (May 2006) 365-372

4. Townend, P., Groth, P., Xu, J.: A provenance-aware weighted fault tolerance scheme for service-based applications. In: 8th IEEE Intl Symp on Object-oriented Real-time Distributed Computing, USA (May 2005) 258-266

5. Foster, I., Voeckler, J., Wilde, M., Zhao, Y.: Chimera: A virtual data system for representing, querying, and automating data derivation. In: 14th Intl Conf on Scientific and Statistical Database Management. (July 2002) 37-46

6. Rajbhandari, S., Walker, D.W.: Support for provenance in a service-based computing Grid. In: UK e-Science All Hands Meeting 2004, UK (September 2004)

7. Zhao, J., Goble, C., Greenwood, M., Wroe, C., Stevens, R.: Annotating, linking and browsing provenance logs for e-Science. In: Wksp on Semantic Web Technologies for Searching and Retrieving Scientific Data (in conjunction with ISWC2003, CEUR Workshop Proceedings). Volume 83., Florida, USA (October 2003)

8. Bose, R., Frew, J.: Composing lineage metadata with XML for custom satellitederived data products. In: 16th Intl Conf on Scientific and Statistical Database Management, Washington, DC, USA (2004) 275-284

9. Simmhan, Y.L., Plale, B., Gannon, D.: A framework for collecting provenance in data-centric scientific workflows. In: IEEE Intl Conf on Web Services 2006 (ICWS 2006), Chicago, USA (September 2006)

10. Simmhan, Y.L., Plale, B., Gannon, D.: A survey of data provenance in e-Science. SIGMOD Record 34(3) (September 2005) 31-36

11. Groth, P., Jiang, S., Miles, S., Munrow, S., Tan, V., Tsasakou, S., Moreau, L.: An architecture for provenance systems. Technical report, Electronics and Computer Science, University of Southampton (October 2006)

12. Feng, Y.H., Cai, W.T.: MCCF: A distributed Grid job workflow execution framework. In: 2nd Intl Symposium on Parallel and Distributed Processing and Applications. Volume 3358., Hong Kong, China, LNCS (December 2004) 274-279

13. Brandt, R., Reiser, H.: Dynamic adaptation of mobile agents in heterogeneous environments. In: 5th Intl Conf on Mobile Agents (MA2001). Volume 2240., Atlanta, USA, LNCS (December 2001) 70-87

14. Fuggetta, A., Picco, G.P., Vigna, G.: Understanding code mobility. IEEE Trans on Software Engineering 24(5) (1998) 342-361

15. Feng, Y.H., Cai, W.T., Cao, J.N.: Communication partner identification in distributed job workflow execution over the Grid. In: 3rd Intl Wksp on Mobile Distributed Computing (in conjunction with ICDCS05), Columbus, Ohio, USA (June 2005) 587-593

16. Cabri, G., Leonardi, L., Zambonelli, F.: Coordination infrastructures for mobile agents. Microprocessors and Microsystems 25(2) (April 2001) 85-92

17. Berger, M.: Multipath packet switch using packet bundling. In: High Performance Switching and Routing (Workshop on Merging Optical and IP Technologies), Kobe, Japan (2002) 244-248

18. Cao, J.N., Zhang, L., Feng, X., Das, S.K.: Path pruning in mailbox-based mobile agent communications. J. of Info Sci and Eng 20(3) (2004) 405-242 